\title{
Influence of Perinatal Risk Factors on Premature Labor Outcome
}

\author{
Agamurad A. Orazmuradov, Phd, $\mathrm{ScD}^{1}$; Mukhamednazar A. Soyunov, $\mathrm{PhD}, \mathrm{ScD}^{1}$; \\ Zhanna M. Efendieva ${ }^{1}$; Aleksey A. Lukaev, MD²*; Irena E. Gradova, MD³ \\ Aiunur V. Mukhtarova, $\mathrm{PhD}^{3}$; Irina N. Kaplina, $\mathrm{MD}^{3}$ \\ ${ }^{1} R U D N$ University, Moscow, Russia \\ ${ }^{2}$ Mytishchi municipal clinical hospital, Mytishchi, Moscow Region, Russia \\ ${ }^{3}$ Municipal clinical hospital № 29 named after N.E. Bauman, Moscow, Russia
}

\begin{abstract}
In this article, for the first time, the problem of premature labor (PL) is considered from the standpoint of the concept of perinatal obstetric risk. The obtained results show that the optimal choice of the mode of delivery must be based on gestational age and perinatal risk factors with calculation of their intrapartum gain (IG). (Int J Biomed. 2016;6(3):199-201.).
\end{abstract}

Key Words: premature labor • perinatal risk factors • perinatal mortality $\bullet$ Cesarean section.

\section{Introduction}

Each year, an estimated 15 million infants are born prematurely, ${ }^{[1]}$ and this number is rising. Complications from preterm birth (PB) are the leading global cause of perinatal mortality (PNM) in developed countries. Prematurity takes the first place in the incidence of PNM: up to $60-70 \%$ of early neonatal mortality and $70-75 \%$ of infant mortality. Stillbirth at premature labor (PL) is 8 to 13 times higher than with labor at term. ${ }^{[2]}$ According to WHO and UNICEF, the number of children under 5 years of age dying each year declined from more than 12.7 million in 1990 to 5.9 million in $2015,{ }^{[3]}$ but among children under 5 years of age, $\mathrm{PB}$ complications are the leading cause of death, responsible for nearly 1 million deaths in 2013. ${ }^{[4]}$ Reducing the burden of PB requires effective maternal care including comprehensive obstetric care (with caesarean section, if needed). Questions about the delivery method in PL are relevant and debated.

In the Russian Federation, there are not clear guidelines and indications for a surgical delivery by Cesarean section (C-section) in PL. National guidelines in obstetrics published with the participation of leading specialists of the Russian Federation recommend individually determining

"Corresponding author: Aleksey A. Lukaev, MD. Mytishchi municipal clinical hospital, Mytishchi, Moscow Region, Russia E-mail: alekseilukaev@mail.ru the indications for abdominal delivery in PL. ${ }^{[5]}$ There is no guidance on accounting for the degree of PR in choosing a method for early delivery in PL. ${ }^{[6]}$

The aim of this study was to improve perinatal outcomes of PL based on the assessment of PR factors and a differentiated selection of mode of delivery.

\section{Materials and Methods}

The study was performed in Municipal clinical hospital № 29 named after N.E. Bauman. We carried out a prospective analysis of 236 medical records of pregnant women with PL at 28 to 33 weeks (plus 6 days) of gestation (code ICD X O60). The study was conducted in accordance with ethical principles of the Declaration of Helsinki.

According to PL classification, all pregnant women were divided into 2 cohorts: Cohort I (gestational age from 28 to 30 weeks plus 6 days) and Cohort II (gestational age from 31 to 33 weeks plus 6 days). Depending on the amount of PR factors, each cohort was divided into 3 groups: a low PR, a moderate PR, and a high PR.

We identified the degree of PR based on the scale developed by O.G. Frolova and E.I. Nikolaeva (1981) and modified in 2003 by V.E. Radzinsky et al..$^{[7]}$ The scale includes a number of parameters divided into blocks: socio-biological block, data of obstetric and gynecological history, extragenital diseases (EGD) of mother, complications of pregnancy, and fetal assessment. 
A specific group for PR was defined in accordance with the number of points derived from S. Knyazev's scale (2003): low risk ( $<15$ points), moderate risk (from 15 to 20 points), and high risk ( $\geq 25$ points). Calculation of PR factors was performed twice: at admission and during labor. The ratio of these indexes determines the so-called "intrapartum gain" (IG) of PR factors. ${ }^{[6]}$

Inclusion criteria were singleton pregnancy, $\mathrm{PB}$ (gestational age from 28 to 33 weeks plus 6 days), and no treatment for cervical insufficiency.

Exclusion criteria were polycyesis, congenital malformations of the fetus revealed during currently pregnancy and after childbirth, induced $\mathrm{PB}$, the use of assisted reproductive technologies, and the scar on the uterus.

In accordance with the purpose of the study, we designed a specific questionnaire for the clinical assessment of the state of health of the pregnant women.

Statistical analysis was performed using the statistical software «Primer of Biostat 4.0» and «STATISTICA 7». Group comparisons with respect to categorical variables are performed using chi-square tests with Yates correction. A probability value of $P<0.05$ was considered statistically significant.

\section{Results and Discussion}

Comparative clinical and statistical analysis of the age and anthropometric indicators, social and marital status, and status of somatic and reproductive health of surveyed pregnant women showed that the two cohorts were comparable, according to analyzed parameters.

The average age of patients was $29.1 \pm 0.4$ years. Overall, $126(53.4 \%)$ of the women were aged between 21 and 30 years, and $72(30.5 \%)$ between 31 and 35 years.

Analyzing the complications of the first trimester of gestation, we revealed the following features. It is well known that smoking increases the risk of PB. ${ }^{[8,9]}$ Our study showed that $103(43.6 \%)$ women were smokers. Moreover, the premature term was significantly higher in smokers $(P<0.05)$. In both cohorts, we revealed moderate correlations between smoking and $\mathrm{PB}(\mathrm{r}=0.32, P<0.05$ and $\mathrm{r}=0.37, P<0.05)$. Pregnant women with a high PR were smokers more often than were pregnant women with low/moderate PR $(P<0.05)$. We found a moderate direct correlation between the degree of $\mathrm{PR}$ and smoking $(\mathrm{r}=0.62, P<0.05)$.

The overall rate of extragenital diseases (EGD) was $100 \%$. In Cohort II, the incidence of EGD was the highest in women with a high PR (8[33.3\%]) compared to women with a moderate PR (6 [11.5\%]) (Yates' $P=0.04998)$ and a low PR (3 [9.4\%]) (Yates' $P=0.05830$ ). Diseases of the genitourinary system (chronic cystitis, pyelonephritis, urolithiasis) were diagnosed in 48 (20.3\%) women without significant differences between groups. Diseases of the genitourinary system were diagnosed in every third pregnant woman with moderate to high PR in Cohort II. There was a moderate correlation between the PR degree and diseases of the genitourinary system in Cohort II $(\mathrm{r}=0.43, P<0.05)$ and a less pronounced correlation for Cohort I $(\mathrm{r}=0.31, P<0.05)$.
Among 236 pregnant women, every ninth woman had obesity $(25[10.6 \%])$, and every third woman $(76[32.2 \%])$ was underweight. Underweight women were identified significantly more often in groups with a high PR.

The study of reproductive health showed that an inflammatory disease of the body of the uterus and the uterine appendages occurred in $43(18.2 \%)$ pregnant women. In Cohort II, these diseases were diagnosed significantly more often in women with a high PR compared to women with moderate to low PR (7 [29/2\%] versus 8 [9.5\%]) (Yates' $P=0.03405)$. Recurrent inflammatory diseases of the external genitalia and vagina occurred in 87 (36.9\%) women. Around 44.3\% and $58.3 \%$ of women (Cohort I and Cohort II, respectively) with a high PR had history of vulvovaginitis. In Cohort II, sexually transmitted infections (STIs) were diagnosed significantly more often in women with a high PR compared to women with low to moderate PR (8 [33.3\%] vs. $11[13.1 \%], P=0.04632)$.

Evaluation of the parity status showed that the number of primigravida women was significantly higher in the low PR groups. Three out of four pregnant women with a high PR were multigravida and gave birth for the first time. In women of both cohorts with a low PR, the number of abortions was lower than in women with moderate to high PR $(1.6 \pm 0.4$ and $1.9 \pm 0.3$, respectively). PL history in 15 (9.1\%) pregnant women showed that almost half of them were in the high PR groups $(46.7 \%)$.

The frequency of a complicated course of pregnancy was $100 \%$. The first trimester and the beginning of the second trimester of gestation is an essential period for the further course of pregnancy and, therefore, affects the prognosis of the perinatal outcomes. This period was characterized by the threat of spontaneous abortion in the majority of patients $(60.6 \%)$. Continuous threat of pregnancy termination, requiring repeated hospital treatment (the average rate of hospitalizations was $2.1 \pm 0.9)$, was diagnosed in $59(25 \%)$ pregnant women; most of these women $(43[72.9 \%])$ had a high PR. Almost every second woman had one abortion in anamnesis; $78 \%$ and $47.6 \%$ of women had two or more abortions. In Cohort II, cervical insufficiency was found in 6 (25.0\%) women with a high PR and $7 / 8.3 \%$ women with low to moderate PR (Yates' $P=0.06325$ ).

In the structure of the pathology of the amniotic fluid, oligohydramnios (45.3\%) was predominant. Oligohydramnios was diagnosed 2 to 3 times more often in women with high to moderate PR compared to women with a low PR. The incidence of oligohydramnios significantly increased with gestational age $(\mathrm{r}=0.547, P<0.01)$. Polyhydramnios was found in $30(12.7 \%)$ women.

Preeclampsia was diagnosed in 43 (18.2\%) women and was a frequent complication in pregnant women with a high PR in Cohort II: $13(54.2 \%)$ vs. $2(6.2 \%)$ pregnant women with a low PR $(P=0.0001)$.

Anemia of pregnancy was diagnosed in 108 (48\%) women without significant differences between cohorts and was more common with increasing gestational age.

PL was often associated with a premature rupture of membranes (63\%) without an association with gestational age. Tocolytic therapy was administered to all pregnant women, 
including an intrapartum tocolysis. The duration of tocolysis in Cohort I was significantly longer than in Cohort II $(P<0.05)$, apparently motivated by the desire to prolong the pregnancy as much as possible.

Vaginal birth occurred in $154(65.3 \%)$ women. C-section was performed in $82(34.7 \%)$ patients: $16(19.5 \%)$ women with a low PR, $32(39.0 \%)$ - a moderate PR and $34(41.5 \%)$ - a high PR.

Fetometry showed that intrauterine growth retardation (IUGR) was diagnosed in 71(30.1\%) patients. The main share of women with IUGR was presented by those with a high PR $(38.0 \%)$ in Cohort I and those with a moderate PR (23.9\%) in Cohort II. Diagnosis of IUGR was confirmed in only $16(22.5 \%)$ newborns (Table 1), and every second newborn was born to a mother of Cohort II with a high PR.

\section{Table 1.}

Analysis of IUGR frequency

\begin{tabular}{|c|c|c|c|c|c|}
\hline \multirow{2}{*}{ ن̃ } & \multirow[t]{2}{*}{ Degree of PR } & \multicolumn{2}{|c|}{$\begin{array}{l}\text { Women diagnosed } \\
\text { with IUGR }\end{array}$} & \multicolumn{2}{|c|}{ IUGR infants } \\
\hline & & $\mathrm{n}(\%)$ & Statistics & $\mathrm{n}(\%)$ & Statistics \\
\hline \multirow{4}{*}{ I } & Low $(n=20)$ & $3(15.0)$ & \multirow{3}{*}{$\begin{array}{c}\chi^{2}=0.397 \\
P=0.81996\end{array}$} & $1(33.3)$ & \multirow{3}{*}{$\begin{array}{c}\text { Yates' } \chi^{2}=0.28 \\
P=0.86936\end{array}$} \\
\hline & Moderate $(\mathrm{n}=38)$ & $8(21.1)$ & & $2(25.0)$ & \\
\hline & $\operatorname{High}(\mathrm{n}=70)$ & $12(17.1)$ & & $4(33.3)$ & \\
\hline & $n=128$ & $23(18.0) *$ & & $7(30.4)$ & \\
\hline \multirow{4}{*}{ II } & Low $(n=32)$ & $17(53.1)$ & \multirow{3}{*}{$\begin{array}{c}\chi^{2}=2.178 \\
P=0.33655\end{array}$} & $2(11.8)$ & \multirow{3}{*}{$\begin{array}{c}\text { Yates' } \chi^{2}=3.65 \\
P=0.16098\end{array}$} \\
\hline & Moderate $(\mathrm{n}=52)$ & $23(44.2)$ & & $3(13.0)$ & \\
\hline & High $(n=24)$ & $8(33.3)$ & & $4(50.0)$ & \\
\hline & $n=108$ & $48(44.4) *$ & & $9(18.8)$ & \\
\hline Total & 236 & $71(30.1)$ & & $16(22.5)$ & \\
\hline
\end{tabular}

* chi-square $=19.521$ and $P=0.0000$ between two cohorts

Disorders in the uteroplacental and fetal-placental circulation were detected in $79(33.5 \%)$ of pregnant women ( 2 and 3 degree in 16[20.2\%] women). These disorders were most common in groups of women with a moderate PR.

Analysis of PNM showed that $8(3.39 \%)$ newborns died, including $6(75.0 \%)$ newborns born to mothers at 28 to 30 weeks (plus 6 days) of gestation. In Cohort 1, the vast majority of the deceased newborns $(66.7 \%)$ were born to mothers with a moderate PR that was significantly more than in other groups $(P<0.05)$.

In Cohort 2, two newborns died; they were born to mothers with moderate to high PR. These data confirm a known fact: PNM decreases with increasing gestational age.

A retrospective analysis of PNM according to the revised risk factors, taking into account their IG, showed another distribution of the analyzed indicators. After recalculation of PR factors with regard to IG, we found that the vast majority of deceased newborns $(87.5 \%)$ were born to mothers with a high PR. In this regard, we have analyzed the critical threshold of IG for PR factors that affected the perinatal outcomes.
It was found that 6 of 8 deceased newborns were born vaginally and belonged to groups of low and moderate PRs. The decision for vaginal delivery was based only on PR factors, despite the fact that the women were patients with moderate and high PRs, according to IG. In general, if the situation with the choice of delivery method in pregnant women with a high PR is very clear, namely, a planned en caul (within intact membranes), C-section is the method of choice for delivery in PL at 28-33 weeks (plus 6 days) of gestation, the choice of mode of delivery in women with moderate to low $\mathrm{PR}$ is ambiguous.

After recalculation of PR factors with regard to their IG, all pregnant women $(100 \%)$ with a low PR was transferred into moderate PR groups; every second pregnant woman (47[52.2\%]) with a moderate PR was transferred into high PR groups. The remaining women $(43[47.8 \%])$ were still in moderate PR groups. Analysis of perinatal mortality and morbidity has demonstrated the presence of statistically significant differences $(P<0.05)$ in the selection of the priority mode of delivery in women with PL at all stages of gestation, depending on PR. Thus, the perinatal mortality and morbidity rate was lower among pregnant women with a high PR who gave birth to premature babies delivered by $\mathrm{C}$-section.

The optimal choice of method of delivery based on PR factors and IG is a resource for reducing perinatal mortality and morbidity. Risk strategy must involve a dynamic recalculation of PR factors, IG, in childbirth.

\section{Competing interests}

The authors declare that they have no competing interests.

\section{References}

1. Nour NM. Premature delivery and the millennium development goal. Rev Obstet Gynecol. 2012; 5(2):100-5.

2. Radzinsky VE. Obstetric aggression. M.: StatusPraesens; 2011, 668 pp. [in Russian].

3. UNICEF website: Levels \& Trends in Child Mortality. Report 2015. http://www.data.unicef.org/corecode/uploads/ document6/uploaded_pdfs/corecode/IGME-report-2015child-mortality-final_236.pdf

4. WHO website: Preterm birth. Fact sheet $N^{\circ} 363$ Updated November 2015. http://www.who.int/mediacentre/factsheets/ fs $363 / \mathrm{en} /$

5. Obstetrics: National Guidelines. G.M. Savel'eva, V.N. Serov, VE Radzinsky (Eds.). Second Edition, Revised and Enlarged. M.: GEOTAR-Media; 2015, 1080 pp. [in Russian]. 6. Orazmuradov AA, Lukaev AA, Yanin VA, Krikunova VL, Verstat TV. Outcomes of Premature Birth in Women Having High Factors for Perinatal Risks. Int J Biomed. 2014;4(1):32-5. 7. Radzinsky VE, Knyazev CA, IN Kostin. Obstetric risk. Maximum of information - Mminimum of danger to the mother and baby. M.: Eksmo; 2009, 290 pp. [in Russian].

8. Kyrklund-Blomberg NB1, Granath F, Cnattingius S. Maternal smoking and causes of very preterm birth. Acta Obstet Gynecol Scand. 2005;84(6):572-7.

9. Dekker GA1, Lee SY, North RA, McCowan LM, Simpson NA, Roberts CT. Risk factors for preterm birth in an international prospective cohort of nulliparous women. PLoS One. 2012;7(7):e39154. 